EXTENDED REPORT

\title{
Chorioretinal temperature monitoring during transpupillary thermotherapy for choroidal neovascularisation
}

\author{
S Miura, H Nishiwaki, Y leki, Y Hirata, Y Honda, Y Sugino, Y Okazaki
}

Br J Ophthalmol 2005;89:475-479. doi: 10.1136/bjo.2004.049189

See end of article for authors' affiliations

....................

Correspondence to: Hirokazu Nishiwaki, $M D$, Department of Ophthalmology and Visual Sciences, Kyoto University Graduate School of Medicine, 54

Shogoinkawahara-cho, Sakyo-ku, Kyoto 6068507, Japan; nishiwak@ kuhp.kyoto-u.ac.jp

Accepted for publication 1 September 2004

\begin{abstract}
Aims: To investigate the difference in temperature rise between normal choroid and choroidal revascularisation (CNV) during transpupillary thermotherapy (TTT) and the relation between laser spot size and power in the rat fundus.

Methods: A modified slit lamp, which was installed with two laser wavelengths (490 nm for illumination and fluorescein excitation and $810 \mathrm{~nm}$ for hyperthermia), was developed for TTा and temperature monitoring. Temperature rise during TTT was monitored by observing fluorescence released from thermosensitive liposomes encapsulating carboxyfluorescein. Two types of liposomes were prepared; their phase transition temperatures were $40^{\circ} \mathrm{C}$ and $46^{\circ} \mathrm{C}$, respectively. Laser power settings required to observe fluorescence released from $46^{\circ} \mathrm{C}$ liposome in normal choroid or CNV were compared. Next, the power settings with $0.5 \mathrm{~mm}$ and $0.25 \mathrm{~mm}$ spot sizes were compared following administration of $40^{\circ} \mathrm{C}$ liposome or $46^{\circ} \mathrm{C}$ liposome.

Results: The minimum power values when release from $46^{\circ} \mathrm{C}$ liposome was observed showed a significant difference in distribution of power values between normal choroid and CNV. CNV required significantly higher power than normal choroid. With $40^{\circ} \mathrm{C}$ liposome, the power was 9.7 (1.9) $\mathrm{mW}$ (mean (SD)) at a spot size of $0.25 \mathrm{~mm}$, and $12.1(1.6) \mathrm{mW}$ at $0.5 \mathrm{~mm}$, respectively. When using $46^{\circ} \mathrm{C}$ liposome, the power setting was $10.2(1.2) \mathrm{mW}$ at a spot size of $0.25 \mathrm{~mm}$, and $14.6(2.2) \mathrm{mW}$ at $0.5 \mathrm{~mm}$, respectively.

Conclusions: CNV demonstrated varying heat conduction, compared with normal choroid. Laser power required to raise the temperature should not necessarily be doubled, even when the spot size is doubled. Close attention should be given to the selection of power settings when performing TTT for CNV.
\end{abstract}

A ge related macular degeneration is the leading cause of central visual loss in patients older than 50 years of age in the United States. ${ }^{12}$ The majority of eyes have severe visual loss as a result of choroidal neovascularisation (CNV), which is the formation of new blood vessels either between the retinal pigment epithelium (RPE) and Bruch's membrane or RPE and subretinal space. The only proved effective treatment is laser photocoagulation. The Macular Photocoagulation Study ${ }^{3}$ demonstrated that treatment of well defined subfoveal CNV was beneficial, but most patients experienced an immediate decline in vision because of damage to the overlying sensory retina.

Transpupillary thermotherapy (TTT) has recently emerged as an advance for treating occult choroidal neovascularisation $(\mathrm{CNV}) .{ }^{4}$ Initially used for the treatment of choroidal melanoma, ${ }^{5}$ TTT is a technique in which heat is delivered to the choroid and retinal pigment epithelium (RPE) through the pupil using a modified laser diode (usually $810 \mathrm{~nm}$ ) delivered from the ophthalmic slit lamp. TTT for CNV is a subthreshold photocoagulation procedure. If retinal whitening is observed, treatment should be stopped, because the goal of treatment is to deliver laser energy to CNV while sparing overlying retina. In contrast with conventional laser photocoagulation, ${ }^{6}$ in which an estimated retinal temperature rise of $42^{\circ} \mathrm{C}$ occurs, the estimated retinal temperature elevation with TTT is calculated at approximately $10^{\circ} \mathrm{C} .{ }^{7}$ Reichel et al reported that, after TTT, 94\% of CNV showed clinical or angiographic improvement and vision was stabilised or improved in $75 \% .^{8}$ Miller-Rivero reported 26 of 30 eyes treated with TTT demonstrated a decrease in exudation after TTT. ${ }^{9}$ These earlier reports have suggested that TTT can result in closure of subfoveal CNV, with relative sparing of the overlying neurosensory retina, compared to conventional laser photocoagulation therapy.
Mainster and Reichel recommended ${ }^{7}$ (1) adjusting the power of TTT based on the fundus environment such as media clarity, degree of pigmentation, RPE atrophy, and choroidal circulation, and (2) doubling or halving the power if spot size is doubled or halved, because the power needed to produce a particular retinal temperature rise is roughly proportional to the diameter rather than the area of laser spot on the retina. In several clinical studies ${ }^{10}{ }^{11}$ concerning TTT, treatment was thought to be performed according to the recommendations above. However, complications caused by overcoagulation have been reported. Although Matsumoto and Yuzama performed TTT in Japanese patients with 50\% of power suggested by Reichel et al, excessive coagulation was observed in two of 48 eyes. ${ }^{10}$ Auer and Herbort reported that choroidal atrophy, which might be caused by overcoagulation was observed after TTT in five of 38 eyes. ${ }^{11}$ These reports suggested that a uniform irradiation setting can lead to the difference in treatment effects among patients.

However, it may be difficult to deliver the appropriate irradiation of TTT in every case, because we have no way to measure chorioretinal temperature rise directly. Thermal dosimetry techniques, subretinal or episcleral microthermocouple, ${ }^{12}$ and magnetic resonance imaging $(\mathrm{MRI})^{13}$ have been reported. At present, however, it may be difficult to apply these techniques to chorioretinal temperature monitoring, since the former is invasive and the latter requires specially adapted and initially costly devices. Because temperature monitoring devices are lacking, there has been no clinical

Abbreviations: CF, carboxyfluorescein; CNV, choroidal revascularisation; FAG, fluorescein angiography; LTM, liposomal temperature monitoring; MRI, magnetic resonance imaging; RPE, retinal pigment epithelium; Tc, phase transition temperatures; TIT, transpupillary thermotherapy 
examination of thermal features of CNV through subthreshold thermotherapy. Thus, appropriate treatment methods for TTT, such as power, duration, and spot size are still controversial. ${ }^{14} 15$

We previously introduced a non-invasive technique to monitor chorioretinal temperature during TTT, the liposomal temperature monitoring (LTM) technique. ${ }^{16}$ Thermosensitive liposomes, vesicles of lipid bilayers, were shown to entrap carboxyfluorescein (CF) at a high concentration and release their contents when liposomes are warmed above their phase transition temperatures (Tc). LTM consisted of applying TTT on the fundus following intravenous injections of thermosensitive liposomes encapsulating CF (CF liposomes). The background fluorescein intensity before and after TTT was low, because the circulating CF liposomes were invisible in the angiograms as a result of self quenching. However, once TTT was started and tissue temperature reached the Tc of injected liposomes, the excitation argon laser in the slit lamp brightly visualised the bolus of CF released from liposomes at the heated lesion. Temperature rise during TTT was monitored by observing the dye front from CF liposomes circulating in the heated tissues.

In this study, with LTM, we investigated features of temperature rise in the rat fundus during TTT. Firstly, we compared the TTT power required to raise tissue temperature to threshold temperature $\left(46^{\circ} \mathrm{C}\right)$ between the normal choroid and CNV. Secondly, we investigated the relation between the spot size and the power with a rise in temperature.

\section{MATERIALS AND METHODS}

\section{Animal anaesthesia and induction of CNV}

Male Long-Evans (LE) rats, weighing 180-200 g each, were used for this study. Twenty four rats were used to perform TTT on normal choroid and 10 rats were used to perform TTT on CNV. The animals were treated in compliance with the ARVO statement for the use of animals in ophthalmic and vision research. The rats were anaesthetised with intramuscular ketamine $(10 \mathrm{mg} / \mathrm{kg})$ and xylazine $(4 \mathrm{mg} / \mathrm{kg})$. Topical $1 \%$ tropicamide and $2.5 \%$ phenylephrine hydrochloride were instilled for mydriasis during laser photocoagulation and TTT.

Dye laser irradiation (545 nm; argon dye laser model 920; Coherent Medical Laser, Canada) was delivered through a slit lamp (Carl Zeiss, Germany) with a hand held 90 dioptre lens (Nikon, Japan). A contact lens was used to retain corneal clarity throughout photocoagulation. Laser spots were placed separately, five burns in each eye, using a setting of $100 \mu \mathrm{m}$ in diameter, for 0.1 second duration, at $150 \mathrm{~mW}$ intensity.

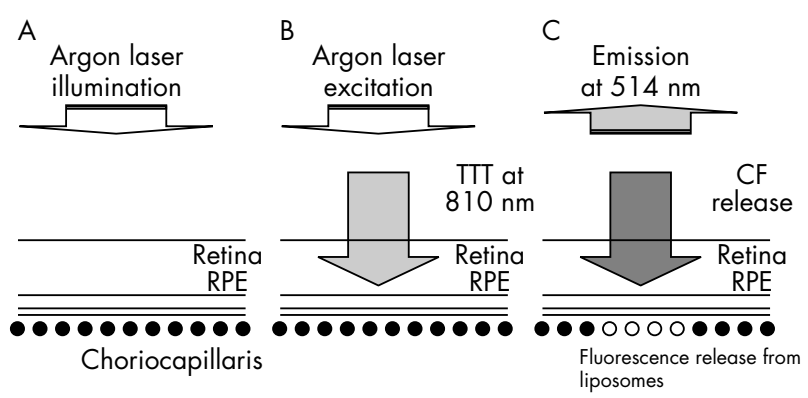

Figure 1 Principle of liposomal temperature monitoring (LTM). (A) Immediately after injection of CF liposome, an illuminating argon laser was delivered to the rat fundus. (B) TाT started. No release of fluorescent bolus was observed, when the temperature increase was below Tc in the heated lesion. (C) The TTT power setting was raised to an adequate value. When the temperature in the lesion was above the Tc of the injected liposome, the emitted fluorescent bolus was observed in and around the heated lesion.
CNV was evaluated on day 14 by ophthalmoscopy, fundus photography, and conventional fluorescein angiography. ${ }^{17}$

\section{CF liposome preparation}

Dipalmitoylphosphatidylcholine (DPPC), dipalmitoylphosphatidylglycerol (DPPG), distearoylglycerophosphocholine (DSPC) (Genzyme, Liestal, Switzerland), and myristoylpalmitoylphosphatidylcholine (MPPC) (Avanti, Alavaster, AL, USA) were used without further purification. Liposomes were prepared by the method previously described.$^{18-21}$ Briefly, a $100 \mathrm{mM}$ solution of 5,6-CF (Molecular Probes, Junction City, OR, USA) was mixed with the dried lipid film, and the mixture was subjected to five cycles of freezing and thawing. This process was followed by extrusion sizing in a thermobarrel extruder (Lipex Biomembranes, Vancouver, Canada) through a stack of two $25 \mathrm{~mm}, 0.2 \mu \mathrm{m}$ polycarbonate membranes (Millipore, Bedford, MA, USA) to yield large, unilamellar vesicles. Unentrapped CF was removed through a Sephadex G-50 column (Pharmacia Biotech, Uppsala, Sweden).

Two types of liposomes were prepared as follows:

(1) $40^{\circ} \mathrm{C}$ liposome $\left(\mathrm{TC}=40^{\circ} \mathrm{C}\right)$, DSPC:DPPC:DPPG:MPPC $=0: 16: 3: 1(\mathrm{~mol} / \mathrm{mol})$

(2) $46^{\circ} \mathrm{C}$ liposome $\left(\mathrm{Tc}=46^{\circ} \mathrm{C}\right)$, DSPC:DPPC:DPPG:MPPC $=47: 43: 10: 20(\mathrm{~mol} / \mathrm{mol})$.

Release yield versus suspension temperature in vitro The amount of CF released was assayed by measuring fluorescence with a spectrofluorophotometer (Shimadzu, Kyoto, Japan) at $490 \mathrm{~nm}$ (excitation) and $514 \mathrm{~nm}$ (emission). A liposome suspension of $30 \mu \mathrm{l}$ was mixed with $3 \mathrm{ml}$ of $50 \%$ human serum. Next, $0.1 \mathrm{ml}$ of Triton X-100 (Sigma, St Louis, MO, USA), which disrupts the vesicles and releases the entrapped $\mathrm{CF}$, was added to the control samples at room temperature. The samples were heated in the water bath at different temperatures for 1 minute. The percentage of CF release was calculated by comparing the fluorescent values of heated samples with total release of CF obtained after the addition of Triton X-100 to the control sample.

\section{Slit lamp modification for TTT}

We modified a slit lamp for TTT, based on the commercial model of SL-10L (Topcon, Tokyo, Japan). The illumination of the slit lamp was switched to an argon laser at $490 \mathrm{~nm}$ (Novus 2000, Coherent, Palo Alto, CA, USA), which made it possible to perform the treatment while visualising and diagnosing the lesion with fluorescein angiography (FAG). In addition, a laser diode at $810 \mathrm{~nm}$ (F-System, Coherent, Palo Alto, CA, USA) was installed as aiming beam and to perform TTT. The zoom system allowed the spot diameter of $810 \mathrm{~nm}$

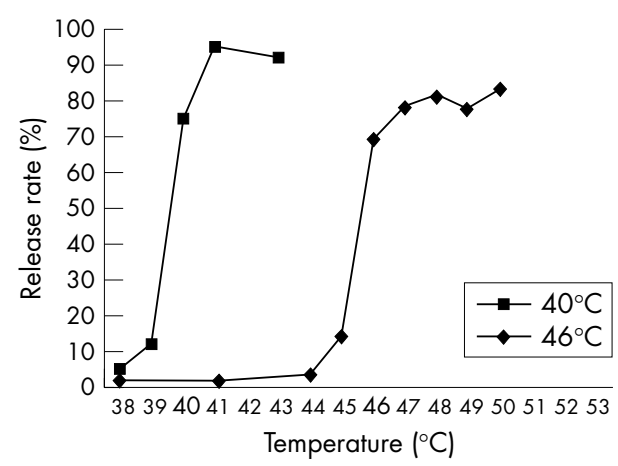

Figure 2 Temperature profiles of CF liposome. Note that CF release rate significantly increased at Tc of each liposome. Squares; $40^{\circ} \mathrm{C}$ liposome, diamonds, $46^{\circ} \mathrm{C}$ liposome. 
laser to be continuously variable $(50-500 \mu \mathrm{m})$. Irradiation time and power could be adjusted using an attached controller. The laser spot was movable with a manipulator. Images taken by a CCD camera (Sony, Tokyo, Japan) equipped on the slit lamp were displayed on a monitor after being amplified by a video enhancer (Seprotec, Tokyo, Japan). The images then were recorded on a digital videotape (Sony, Tokyo, Japan).

\section{Principle of monitoring temperature rise during TTT}

Figure 1 illustrates the principle of liposomal temperature monitoring (LTM). The TTT setting selected for the present study was as follows: exposure time, 60 seconds and power, 0-40 mW. TTT was delivered with the modified slit lamp through a fixed 78 dioptre lens (Volk, Mentor, OH, USA). At the beginning of the experiment (two minutes before TTT), liposome suspension $(1.0 \mathrm{ml} / \mathrm{kg})$ was injected into the rat tail vein. Immediately before TTT, the fundus image was observed with the illuminating argon laser and recorded as background fluorescence (fig 1A). A weak aiming beam at $810 \mathrm{~nm}$ was focused on the target site. Next, we applied TTT with $810 \mathrm{~nm}$ laser to normal choroid or laser induced CNV (fig 1B). The absorbed energy of hyperthermic laser caused the tissues to warm up. When tissue temperatures reached the Tc of injected liposomes, liposomes in the vasculature released their contents. Released CF was excited with the illuminating argon laser at $490 \mathrm{~nm}$, and fluorescent patterns emitted at $514 \mathrm{~nm}$ were observed on the TV monitor and recorded (fig IC). When the fluorescent bolus during TTT demonstrated lobular and "honeycomb" patterns that Asrani et al have reported as choriocapillary vascular patterns, ${ }^{19}$ the choroidal temperature in the heated lesion was considered above the Tc. A contact lens was used to retain corneal clarity throughout treatment.

\section{Comparison of minimum TTT power setting for} temperature rise between normal choroid and CNV After the intravenous injection of $46^{\circ} \mathrm{C}$ liposome, TTT for normal choroid and CNV was performed with a $0.5 \mathrm{~mm}$ spot size through LTM. In rats with normal choroid $(n=16)$ and those with $\mathrm{CNV}(\mathrm{n}=20)$, the power was increased until release of $\mathrm{CF}$ from the $46^{\circ} \mathrm{C}$ liposome was observed, and the power value at the time of observation was recorded. Laser power settings required to observe fluorescence released from liposomes were compared statistically. All values were mean (SD). The F test was used to analyse the variation in the
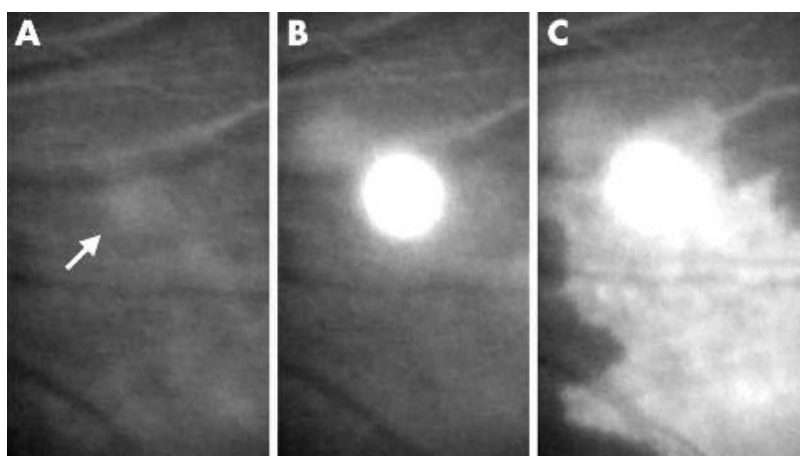

Figure 3 LTM applied to the normal choroid. (A) Background fluorescence. Immediately after injection of CF liposome, an illuminating laser slightly delineated major retinal vessels. However, no fluorescence leaked in the retinal vasculature. Arrow: a weak aiming laser at $810 \mathrm{~nm}$. (B) TTT started after injection of CF liposome. No release of fluorescent bolus was observed, because the temperature rise was inadequate in the heated lesion. (C) When the temperature ruse was above the Tc of the injected liposome, patchy fluorescence around heated lesion was observed. distribution of each group. Welch's $t$ test was used to compare TTT power setting for normal choroid and CNV. Differences were considered statistically significant if $\mathrm{p}<0.05$.

\section{Investigation of relation between spot sizes and TTT power settings in temperature rise}

Following the intravenous administration of $40^{\circ} \mathrm{C}$ or $46^{\circ} \mathrm{C}$ liposomes, the TTT for normal choroid was performed through LTM. With $0.5 \mathrm{~mm}$ or $0.25 \mathrm{~mm}$ spot sizes, the power was increased until release of CF from the liposome was observed, and the power value at the time of observation was recorded. The difference in power settings between $0.5 \mathrm{~mm}$ and $0.25 \mathrm{~mm}$ spot sizes was compared individually in cases given the $40^{\circ} \mathrm{C}$ liposome and $46^{\circ} \mathrm{C}$ liposome with the unpaired $t$ test. Differences were considered statistically significant if $\mathrm{p}<0.05$.

\section{RESULTS}

Release yield versus suspension temperature in vitro The release yield was measured at different temperatures in each liposome, and the results were summarised (fig 2) as temperature profiles. The Tc of each liposome in vitro showed a theoretical value. The CF release rate sharply increased at each Tc. This result indicates that circulating liposomes are invisible in the angiograms because of self quenching because of high CF concentration, but that once they are heated and the temperature reaches their Tc, the liposomes release their contents and the CF dye spread in the bloodstream is excited with the illuminating argon laser.

\section{LTM angiograms during TTT}

Figure 3 shows angiographic images when LTM was delivered to the rat fundus. After injection of the CF liposome suspension, no fluorescence was observed in the choroid or retina, but retinal major vessels were slightly delineated with the illuminating argon laser (fig 3A). As soon as TTT started, the heated lesion was outlined as a bright white circle during TTT. When the TTT power setting was low, no fluorescent bolus was observed around the TTT spot (fig 3B). However, once the TTT power was raised adequately and the temperature in the spot reached the Tc of the injected CF liposome, CF dye began to spread around the heated lesion and reached a plateau immediately after the start of TTT. The video image (fig 3C) clearly indicated that the $\mathrm{CF}$ release was located on the external retina, because that fluorescence pattern was consistent with the honeycomb reported to be the choriocapillary network. ${ }^{18-20}$ The location of CNV was determined with the illuminating argon laser before TTT, because a slight fluorescent leakage was observed around the

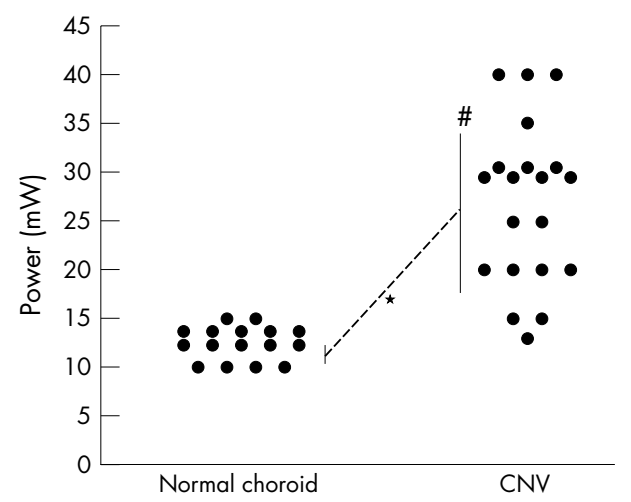

Figure 4 The minimum power values when release of CF from $46^{\circ} \mathrm{C}$ liposome was observed. Note the significant difference in distribution of power values between normal choroid and CNV (\#: $p<0.00001)$. CNV required significantly higher power than normal choroid ( $\left.{ }^{*} p<0.05\right)$. 
CNV. At the start of TTT, a bright white circle that exhibited the heated lesion covered the centre of the CNV lesion. Patchy fluorescence, reported by Asrani et al as choriocapillary pattern, ${ }^{19}{ }^{20}$ began to spread around the CNV and reached a plateau immediately after the start of TTT. It disappeared immediately after TTT ended.

\section{Comparison of minimum TTT power setting for temperature rise between normal choroid and CNV} LTM were performed on normal choroid or CNV with a fixed spot size $(0.5 \mathrm{~mm})$ after injections of $46^{\circ} \mathrm{C}$ liposome suspension. Figure 4 shows the minimum power values when release of $\mathrm{CF}$ from $46^{\circ} \mathrm{C}$ liposome was observed. The $\mathrm{p}$ value of the $\mathrm{F}$ test was $\mathrm{p}<0.0000 \mathrm{l}$, and showed a significant difference in distribution of power values between normal choroid and CNV. This difference in power distribution means power settings to warm $\mathrm{CNV}$ to a particular temperature should have a wide range, compared with normal choroid. In addition, CNV (mean 26.9 (SD 8.3) $\mathrm{mW}$ ) required significantly higher power than normal choroid (12.5 (1.6) mW).

\section{Investigation of relation between spot sizes and TTT power settings in temperature rise}

LTM were performed on normal choroid with a $0.25 \mathrm{~mm}$ or $0.5 \mathrm{~mm}$ spot size, following $40^{\circ} \mathrm{C}$ liposome or $46^{\circ} \mathrm{C}$ liposome. Figure 5 shows the power values when release of CF from $40^{\circ} \mathrm{C}$ liposome and $46^{\circ} \mathrm{C}$ liposome, respectively, was observed. In LTM with $40^{\circ} \mathrm{C}$ liposome, the power was 9.7 (1.9) $\mathrm{mW}$ at a spot size of $0.25 \mathrm{~mm}$, and 12.1 (1.6) $\mathrm{mW}$ at $0.5 \mathrm{~mm}$. When using $46^{\circ} \mathrm{C}$ liposome, the power setting was 10.2 (1.2) $\mathrm{mW}$ at a spot size of $0.25 \mathrm{~mm}$, and $14.6(2.2) \mathrm{mW}$ at $0.5 \mathrm{~mm}$. These results indicated statistically significant differences in TTT power settings between the spot sizes of $0.25 \mathrm{~mm}$ and $0.5 \mathrm{~mm}$.

\section{DISCUSSION}

TTT was initially reported as a hyperthermic technique for treating choroidal melanoma. ${ }^{5223}$ Laser irradiation at $810 \mathrm{~nm}$, which was minimally absorbed by ocular media, was delivered to RPE and tumour melanin. The absorbed laser power produced tissue necrosis within the tumour by causing a temperature rise to levels of approximately $65^{\circ} \mathrm{C}$. The goal of therapy was to achieve a greyish-white colour change in the tumour at the end of TTT.

Recently, TTT was applied to the treatment of CNV as a subthreshold therapy to spare the overlying neurosensory

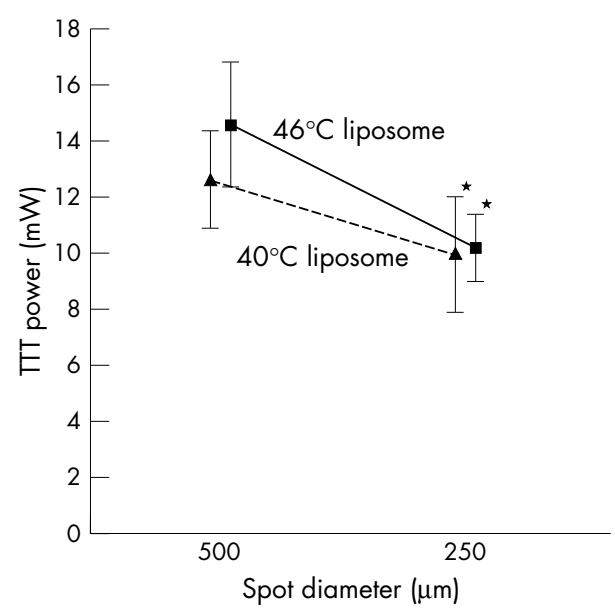

Figure 5 The power values at release of $\mathrm{CF}$ from $40^{\circ} \mathrm{C}$ liposome (triangles) and $46^{\circ} \mathrm{C}$ liposome (squares) was observed ( $\left.{ }^{*} p<0.05\right)$. retina. ${ }^{44-26}$ The tissue temperature in TTT for CNV was calculated to be about $46-47^{\circ} \mathrm{C}^{27}$ which was lower than that for melanomas. In contrast with TTT for melanomas, TTT for $\mathrm{CNV}$ required no or barely visible colour change in the treated lesion to prevent thermal coagulation from occurring in the overlying retina.

Previously, we had introduced a non-invasive technique to monitor chorioretinal temperature during TTT-liposomal temperature monitoring (LTM). ${ }^{16}$ LTM entailed the observation of fluorescence of released CF from thermosensitive liposomes with a modified slit lamp. CF liposomes released capsulated CF effectively when choroid or retina was heated to the levels of their phase transition temperatures (Tc). Released CF produced bright fluorescent images, which signalled to us that tissue temperature in the spot reached the Tc. On the other hand, LTM had some problem when applied to human eyes. Firstly, LTM required an intravenous injection of CF liposome suspension and a specially modified slit lamp. This complexity of procedure could make the application of LTM difficult. Secondly, LTM is indirect temperature monitoring. With LTM, we could just see that the temperature in the irradiated lesion reached or exceeded the Tc of the injected liposome; but we could not measure an arbitrary temperature. However, when we performed LTM, the TTT power setting gradually increased. Thus, the values presented here should be regarded as the best approximation to those values to heat the irradiated lesion to the target temperature (Tc). In addition, our previous report with LTM was consistent with the calculated model. ${ }^{16}$ Thus, LTM was considered to be a reliable method.

In this study with LTM, we observed two findings concerning temperature rise during TTT for normal choroid or CNV. The first finding was that CNV demonstrated varying heat conduction compared with normal choroid. In contrast with TTT on the normal choroid, the power settings to warm $\mathrm{CNV}$ varied individually. We speculated that the form or thickness of CNV, damage to the RPE, or existence of subretinal fluid resulted in a variation of heat conduction in experimental CNV. This feature of varying heat conduction could be applied to clinical CNV, since clinical CNV is often accompanied by subretinal haemorrhage, exudative retinal detachment, retinal oedema, or RPE atrophy. Haemoglobin absorbs visible and infrared irradiation less effectively than melanin. ${ }^{28}$ However, thick haemorrhage can generate significant temperature elevations and prevent optical irradiation from reaching underlying CNV. Subretinal exudation and RPE atrophy would also cause variations in temperature rise across the TTT field. Thus, our results suggested that it would be difficult to obtain an appropriate temperature rise in all patients with TTT under uniform power settings, and it could be necessary to individualise appropriate power settings for each CNV by using a temperature monitoring technique such as LTM.

The second finding was that the laser power required to raise temperature should not necessarily be doubled, even when the spot size is doubled. Theoretically, if the spot size were doubled, doubled power would be necessary to raise temperature. ${ }^{29}$ However, in the experiment with LTM, when the spot size was doubled we observed released fluorescence from $40^{\circ} \mathrm{C}$ or $46^{\circ} \mathrm{C}$ liposome with less power than the theoretical values. We considered two reasons why this result was obtained. Firstly, a small cooling effect by choroidal blood flow $^{30} 31$ might occur in the centre of the spot. Because there were low temperature (that is, body temperature) areas near the peripheral part of the spot, the blood flow of choriocapillaris from these low temperature areas could easily cool the peripheral part. However, since the blood flow into the centre had been already heated in the periphery, the temperature in the centre might easily 
increase. As a result, the centre might be heated by a power that was less than the theoretical value even when the spot size was increased. Secondly, there would be a laser density gradient in the spot. In other words, laser density would decrease more in the periphery, further away from the centre. Under these circumstances, when the spot size was doubled, the target temperature (that is, phase transition temperature of liposome) could be achieved with a power that was less than the calculated one because the centre of the spot, with higher laser density, was heated effectively. This might result in observations of CF released from liposome with a lower power setting than expected.

In conclusion, our results suggested that close attention should be given to the selection of power settings when performing TTT for CNV to accommodate individual difference in temperature rise in CNV. We should adjust TTT power settings to the individual cases. In addition, we should also pay close attention to TTT power settings when we change spot size. Our results suggested that we should not lightly increase the power because appropriate laser power for TTT is not proportional to the spot size.

\section{Authors' affiliations}

S Miura, H Nishiwaki, Y leki, Y Hirata, Y Honda, Department of Ophthalmology and Visual Sciences, Kyoto University Graduate School of Medicine, Kyoto, Japan

Y Sugino, Y Okazaki, Topcon Corporation, Tokyo, Japan

Grant support: Supported by a grant in aid for Scientific Research from the Ministry of Education, Science, and Culture.

Proprietary interest category: None.

\section{REFERENCES}

1 Freund KB, Yannuzzi LA, Sorenson JA. Age-related macular degeneration and choroidal neovascularization. Am J Ophthalmol 1993;1 15:786-91.

2 Ferris FL, Fine SL, Hyman L. Age-related macular degeneration and blindness due to neovascular maculopathy. Arch Ophthalmol 1984;102:1640-2.

3 Macular Photocoagulation Study Group. Age-related macular degeneration. The Macular Photocoagulation Study. Am J Ophthalmol 1984;98:376-7.

4 Newsom RS, McAlister JC, Saeed M, et al. Transpupillary thermotherapy (TTT) for the treatment of choroidal neovascularisation. $\mathrm{Br} J$ Ophthalmol 2001;85:173-8.

5 Keunen JE, Journee-de-Korver JG, Oosterhuis JA. Transpupillary thermotherapy of choroidal melanoma with or without brachytherapy: a dilemma. Br J Ophthalmol 1999;83:1212-13.

6 Mainster MA. Decreasing retinal photocoagulation damage: principles and techniques. Sem Ophthalmol 1999;14:200-9.

7 Mainster MA, Reichel E. Transpupillary thermotherapy for age-related macular degeneration: long-pulse photocoagulation, apoptosis, and heat shock proteins. Ophthalmic Surg Lasers 2001;31:359-73.

8 Reichel E, Berrocal AM, lp M, et al. Transpupillary thermotherapy of occult subfoveal choroidal neovascularization in patients with age-related macular degeneration. Ophthalmology 1999;106:1908-14.
9 Miller-Rivero. Transpupillary thermotherapy in the treatment of occult and classic choroidal neovascularization. Abstract ARVO Invest Ophthalmol Vis Sci 2001;41:S179.

10 Matsumoto $Y$, Yuzawa M. Short-term evaluation and angiography findings in transpupillary thermotherapy. Folia Ophthalmol Jpn 2003;54:578-83.

11 Auer C, Herbort C. Transpupillary thermotherapy for occult subfoveal neovessels in age-related macular degeneration: importance of patient pigmentation for the determination of laser settings. Klin Monatsb/ Augenheilkd 2002;219:250-3.

12 Cain CP, Welch AJ. Measured and predicted laser-induced temperature rises in the rabbit fundus. Invest Ophthalmol 1974;13:60-70.

13 Stollberger R, Ascher PW, Huber D, et al. Temperature monitoring of interstitial thermal tissue coagulation using MR phase images. J Magn Reson Imaging 1998;8:188-96.

14 Thach A, Dugel PU, Sneed SR, et al. Large-spot size transpupillary thermotherapy for the treatment of occult choroidal neovascularization associated with age-related macular degeneration. Arch Ophthalmol 2002;121:817-20.

15 Connolly BP, Regillo CD, Eagle RC Jr, et al. The histopathologic effects of transpupillary thermotherapy in human eyes. Ophthalmology 2003; 110:415-20.

16 Miura S, Nishiwaki $\mathrm{H}$, leki $\mathrm{Y}$, et al. Noninvasive technique for monitoring chorioretinal temperature during transpupillary thermotherapy with a thermosensitive liposome. Invest Ophthalmol Vis Sci 2002;44:2717-21.

17 Kamizuru H, Kimura H, Yasukawa T, et al. Monoclonal antibody-mediated drug targeting to choroidal neovascularization in the rat. Invest Ophthalmol Vis Sci 2001;42:2664-72.

18 Asrani S, Zou S, D'Anna S, et al. Noninvasive visualization of blood flow in the choriocapillaris of the rat. Invest Ophthalmol Vis Sci 1996;37:312-17.

19 Asrani S, Zou S, D'Anna S, et al. Selective visualization of choroidal neovascular membranes. Invest Ophthalmol Vis Sci 1996;37:1642-50.

20 Kiryu J, Shahidi M, Mori MT, et al. Noninvasive visualization of the choriocapillaris and its dynamic filling. Invest Ophthalmol Vis Sci 1994;35:3724-31

21 Hirata Y, Nishiwaki H, Miura S, et al. In vivo analysis of choroidal circulation by continuous laser-targeted angiography in the rat. Invest Ophthalmol Vis Sci 2003;44:3103-9.

22 Robertson DM, Buettner H, Bennett SR. Transpupillary thermotherapy as primary treatment for small choroidal melanomas. Arch Ophthalmol 1999; 117:1512-19.

23 Ip M, Kroll A, Reichel E. Transpupillary thermotherapy. Sem Ophthalmol 1999; 14:11-18.

24 Kaga T, Fonseca RA, Dantas MA, et al. Transient appearance of classic choroidal neovascularization after transpupillary thermotherapy for occult choroidal neovascularization. Retina 2001;21:172-3.

25 Rogers AH, Reichel E. Transpupillary thermotherapy of subfoveal occult choroidal neovascularization. Curr Opin Ophthalmol 2001;12:212-15.

26 Gillies MC, Sarks JP, Beaumont PE, et al. Treatment of choroidal neovascularisation in age-related macular degeneration with interferon alfa2a and alfa-2b. Br J Ophthalmol 1993;77:759-65.

27 Mainster MA, White TJ, Tips JH, et al. Retinal-temperature increases produced by intense light sources. J Opt Soc Am 1970;60:264-70.

28 Mainster MA. Wavelength selection in macular photocoagulation. Tissue optics, thermal effects, and laser systems. Ophthalmology 1986;93:952-8.

29 Mainster MA, Allen RG. Spectral dependence of retinal damage produced by intense light sources. J Opt Soc Am 1970;60:848-55.

30 Parver LM, Auker C, Carpenter DO. Choroidal blood flow as a heat dissipating mechanism in the macula. Am J Ophthalmol 1980;89:641-6.

31 White TJ, Mainster MA, Tips JH, et al. Chorioretinal thermal behavior. Bull Math Biophys 1970;32:315-22. 